\title{
Risk Factors and Altered Parameters in Pregnant Women Infected by Toxoplasma gondii in Lebanon
}

\author{
Farouk Kaakour ${ }^{1}$, Leila Farhat ${ }^{1}$, Widad Dia ${ }^{1} \&$ Seifedine Kadry ${ }^{1}$ \\ ${ }^{1}$ Faculty of Science, Beirut Arab University, Lebanon \\ Correspondence: Seifedine Kadry, Faculty of Science, Beirut Arab University, Beirut, Lebanon. E-mail: \\ s.kadry@bau.edu.lb
}

Received: June 8, 2019 Accepted: September 20, 2019 Online Published: October 8, 2019

doi:10.5539/gjhs.v11n12p65

URL: https://doi.org/10.5539/gjhs.v11n12p65

\begin{abstract}
Toxoplasmosis is a parasitic infection caused by Toxoplasma gondii. Both animals and humans could develop the infection through several ways including diet, unhygienic habits, direct contact to soil, blood transfusions and organ grafting. Although all people are at risk of the infection, ones with weakened immune systems are at much higher risk, out of these are cancer patients, pregnant women, fetuses and newborns. In our study, we collected data from three gynecologists in two Lebanese regions in order to evaluate the prevalence of the infection and the risk factors associated with it. We found out that the increase in age and pet ownership is directly correlated with the number of infected women and the infection of the women is directly related to the mode of delivery being c-section and having infected offspring.
\end{abstract}

Keywords: risk factors, pregnant, toxoplasma

\section{Introduction}

Toxoplasma gondii is a single-celled parasite that causes human and animal toxoplasmosis. It is wide spread, infecting one third of the human population worldwide. Cats belonging to the family Felidae are the only hosts for the sexual stages of this parasite (Jones et al., 2001). T. gondii goes through 3 stages in its host: tachyzoites and bradyzoites which occur in body tissues and sporozoites that get excreted in cats' feces. It can be transmitted between warm blood animals and humans through several routes (Guerina \& Lee, 2017; Hill \& Dubey, 2002). One of the modes of transmission is through ingestion of contaminated fruits, vegetables, raw meat, water and unpasteurized milk in addition to poor hygiene around food and cookware. Another route could be through the direct contact with contaminated soil by gardening and farming or by changing cat litter boxes. Moreover, this parasitic infection could be transmitted through organ transplantation, blood transfusions or open wounds (Soares \& Caldeira, 2019; Olariu et al., 2019). One characteristic of this parasite is its ability to survive in its host for prolonged periods of time. Infected individuals do not show any severe symptoms due to their healthy immune system that is keeping the parasite from causing illness (Bernsteen et al., 1999). However, individuals with compromised immune systems and more especially pregnant women should be aware from this parasite infection since multiple health problems could arise and require immediate assistance (Dun et al., 1999).

In normal cases, toxoplasmosis has flu like symptoms but it is serious in cases of comprised immunity like in chemo-treated patients, newborns and pregnant women. Newly infected mothers with Toxoplasma gondii during or before pregnancy have a high risk of transmitting this parasite to their infants (Smith, 1999). The risk of toxoplasmosis transmission to a fetus increases greatly depending on the gestational age at maternal infection (Dun et al., 1999). Another factor that influences the occurrence of the infection in pregnant women is the high progesterone level that leads to reducing the cell-mediated immune response which is important against the $T$. gondii so the chances of infection in pregnant women fire up (Olariu, 2019).

The purpose of this study is to estimate the prevalence of toxoplasmosis in pregnant Lebanese women and the risk of congenital infection in their fetuses and newborns on the basis of genetic and environmental factors.

\section{Data Collection}

The data was collected over a period of two months in Chouf and Saida regions from three gynecologists, Dr. Tatiana Bou Orm, Dr. Alia Chebbo and Dr. Bouchra El-Hajj. The study aimed at studying the frequency of pregnant women infected by T. gondii, the factors that influenced the infection and the effect on the fetuses and 
newborns of these women. The women in our sample were twenty to forty-five years old. The variables were chosen based on the target of the study which is to check for a relation between the occurrence of ${ }^{\text {the }}$ disease and some genetic, biological and environmental factors. The data was obtained from the patients' files under the Drs'. The variables looked at for the pregnant women were: age, weight, family history, residence, TSH, vit D3, seroprevalence of toxoplasmosis, hemoglobin $(\mathrm{Hgb})$, hepatitis B surface antigen ( $\mathrm{HBsAg}$ ), rubella test, fasting blood sugar level, blood type, week of delivery, method of delivery and pet ownership. As for the babies, we noted their gender and health status. The data was entered into IBM SPSS Software to be organized and summarized in order to clearly analyze it and obtain a solid conclusion.

\section{Results}

Before testing all the variables that may have a relation with toxoplasmosis, we measured the percentage of women having toxoplasmosis in our sample

\subsection{Descriptive Statistics}

\section{Toxoplasmosis infection}

\begin{tabular}{llcccc}
\hline & & Frequency & Percent & Valid Percent & Cumulative Percent \\
\hline \multirow{3}{*}{ Valid } & yes & 53 & 45.3 & 45.3 & 45.3 \\
\cline { 2 - 5 } & no & 64 & 54.7 & 54.7 & 100.0 \\
\cline { 2 - 5 } & Total & 117 & 100.0 & 100.0 & \\
\hline
\end{tabular}

Percentage of woman infected by toxoplasmosis: $45.3 \%$ of pregnant women are infected with toxoplasmosis.

\begin{tabular}{llcccc}
\hline Age & \multicolumn{1}{c}{} & & \\
\hline \multirow{3}{*}{ Valid } & {$[20-30]$} & 26 & Percent & Valid Percent & Cumulative Percent \\
\cline { 2 - 6 } & {$[30-40]$} & 31 & 22.2 & 22.2 & 22.2 \\
\cline { 2 - 6 } & {$[40-50]$} & 60 & 26.5 & 26.5 & 48.7 \\
\cline { 2 - 6 } & Total & 117 & 51.3 & 51.3 & 100.0 \\
\hline
\end{tabular}

Age: $51.3 \%$ of women are of ages between [40-50]

\begin{tabular}{llccc}
\hline Owning a pet & & & & \\
\hline & Frequency & Percent & Valid Percent & Cumulative Percent \\
\hline yes & 62 & 53.0 & 53.0 & 53.0 \\
\cline { 2 - 5 } no & 55 & 47.0 & 47.0 & 100.0 \\
\cline { 2 - 5 } Total & 117 & 100.0 & 100.0 & \\
\hline
\end{tabular}

Owning a pet: $53 \%$ of subjects have pet.

\begin{tabular}{llcccc}
\hline Method of delivery & & & & \\
\hline & & Frequency & Percent & Valid Percent & Cumulative Percent \\
\hline \multirow{3}{*}{ Valid } & Normal & 29 & 24.8 & 24.8 & 24.8 \\
\cline { 2 - 6 } & c section & 88 & 75.2 & 75.2 & 100.0 \\
\cline { 2 - 5 } & Total & 117 & 100.0 & 100.0 & \\
\hline
\end{tabular}

Method of delivery: $75.2 \%$ of subjects delivered by c-section. 


\section{Delivery weeks}

\begin{tabular}{llcccc}
\hline & & Frequency & Percent & Valid Percent & Cumulative Percent \\
\hline \multirow{3}{*}{ Valid } & {$[30-37]$} & 10 & 8.5 & 8.5 & 8.5 \\
\cline { 2 - 6 } & {$[37-40]$} & 84 & 71.8 & 71.8 & 80.3 \\
\cline { 2 - 6 } & {$[40-41]$} & 23 & 19.7 & 19.7 & 100.0 \\
\cline { 2 - 5 } & Total & 117 & 100.0 & 100.0 & \\
\hline
\end{tabular}

Week of delivery: $71.8 \%$ of newborns were delivered between weeks 37 and 40 .

\begin{tabular}{llcccc}
\hline Health status of newborn & & & & \\
\hline & & Frequency & Percent & Valid Percent & Cumulative Percent \\
\hline \multirow{3}{*}{ Valid } & 72 & 61.5 & 61.5 & 61.5 \\
\cline { 2 - 6 } & normal & 45 & 38.5 & 38.5 & 100.0 \\
\cline { 2 - 6 } & Totected & 117 & 100.0 & 100.0 & \\
\hline
\end{tabular}

Health status: $38.5 \%$ of newborns were infected.

\section{Body weight}

\begin{tabular}{llcccc}
\hline & Frequency & Percent & Valid Percent & Cumulative Percent \\
\hline \multirow{5}{*}{ Valid } & 33 & 28.2 & 28.2 & 28.2 \\
\cline { 2 - 6 } & {$[50-65]$} & 43 & 36.8 & 36.8 & 65.0 \\
\cline { 2 - 5 } & {$[65-79]$} & 28 & 23.9 & 23.9 & 88.9 \\
\cline { 2 - 5 } & {$[95-94]$} & 9 & 7.7 & 7.7 & 96.6 \\
\hline$[109-135]$ & 4 & 3.4 & 3.4 & 100.0 \\
\hline
\end{tabular}

Body weight: $36.8 \%$ of subjects' weigh [65-79] kg

\begin{tabular}{llcccc}
\hline Address & \multicolumn{5}{c}{} \\
\hline \multirow{3}{*}{ Valid } & city & 38 & Percent & Valid Percent & Cumulative Percent \\
\cline { 2 - 6 } & village & 79 & 32.5 & 32.5 & 32.5 \\
\cline { 2 - 6 } & Total & 117 & 67.5 & 67.5 & 100.0 \\
\hline
\end{tabular}

Address: $67.5 \%$ of subjects live in villages.

\section{Family history}

\begin{tabular}{llcccc}
\hline & & Frequency & Percent & Valid Percent & Cumulative Percent \\
\hline \multirow{3}{*}{ Valid } & 31 & 26.5 & 26.5 & 26.5 \\
\cline { 2 - 5 } & yes & 86 & 73.5 & 73.5 & 100.0 \\
\cline { 2 - 6 } & Total & 117 & 100.0 & 100.0 & \\
\hline
\end{tabular}

Family history: $26.5 \%$ of women have a family history of toxoplasmosis. 


\section{Rubella infection}

\begin{tabular}{cccccc}
\hline & & Frequency & Percent & Valid Percent & Cumulative Percent \\
\hline \multirow{3}{*}{ Valid } & yes & 88 & 75.2 & 75.2 & 75.2 \\
\cline { 2 - 6 } & no & 29 & 24.8 & 24.8 & 100.0 \\
\cline { 2 - 6 } & Total & 117 & 100.0 & 100.0 & \\
\hline
\end{tabular}

Rubella: $24.8 \%$ of pregnant woman were not affected with rubella.

\section{Hbs Ag}

\begin{tabular}{llcccc}
\hline & & Frequency & Percent & Valid Percent & Cumulative Percent \\
\hline \multirow{2}{*}{ Valid } & yes & 87 & 74.4 & 74.4 & 74.4 \\
\cline { 2 - 5 } & no & 30 & 25.6 & 25.6 & 100.0 \\
\cline { 2 - 5 } & Total & 117 & 100.0 & 100.0 & \\
\hline
\end{tabular}

Hbs Ag: $74.4 \%$ of women have the antigen Hbs Ag.

\section{Hbg}

\begin{tabular}{llcccc}
\hline & & Frequency & Percent & Valid Percent & Cumulative Percent \\
\hline \multirow{3}{*}{ Valid } & {$[7-10]$} & 17 & 14.5 & 14.5 & 14.5 \\
\cline { 2 - 6 } & {$[10-14]$} & 100 & 85.5 & 85.5 & 100.0 \\
\cline { 2 - 5 } & Total & 117 & 100.0 & 100.0 & \\
\hline
\end{tabular}

Hemoglobin level: $85.5 \%$ of subjects had a Hg level between [10-14] g/dl.

\begin{tabular}{llcccc}
\hline Vitamin D3 level & & & & \\
\hline & & Frequency & Percent & Valid Percent & Cumulative Percent \\
\hline \multirow{3}{*}{ Valid } & Deficiency & 100 & 85.5 & 85.5 & 85.5 \\
\cline { 2 - 6 } & Normal & 17 & 14.5 & 14.5 & 100.0 \\
\cline { 2 - 6 } & Total & 117 & 100.0 & 100.0 & \\
\hline
\end{tabular}

Vitamin D3 level: $85.5 \%$ of subjects had vit D3 deficiency.

\section{TSH level}

\begin{tabular}{llrrrc}
\hline & & Frequency & Percent & Valid Percent & Cumulative Percent \\
\hline \multirow{3}{*}{ Valid } & Normal & 115 & 98.3 & 98.3 & 98.3 \\
\cline { 2 - 5 } & Deficiency & 2 & 1.7 & 1.7 & 100.0 \\
\cline { 2 - 5 } & Total & 117 & 100.0 & 100.0 & \\
\hline
\end{tabular}

TSH: $98.3 \%$ of subjects had a normal TSH level.

\section{Gender}

\begin{tabular}{llcccc}
\hline & & & & & \\
& Frequency & Percent & Valid Percent & Cumulative Percent \\
\hline \multirow{3}{*}{ Valid } & male & 75 & 64.1 & 64.1 & 64.1 \\
\cline { 2 - 6 } & female & 42 & 35.9 & 35.9 & 100.0 \\
\cline { 2 - 6 } & Total & 117 & 100.0 & 100.0 & \\
\hline
\end{tabular}

Gender: $64.1 \%$ of newborns were males and 35.9 were females.

\section{blood type}




\begin{tabular}{|c|c|c|c|c|c|}
\hline & & Frequency & Percent & Valid Percent & Cumulative Percent \\
\hline \multirow{9}{*}{ Valid } & A- & 1 & .9 & .9 & .9 \\
\hline & $\mathrm{A}+$ & 31 & 26.5 & 26.5 & 27.4 \\
\hline & AB- & 1 & .9 & .9 & 28.2 \\
\hline & $\mathrm{AB}+$ & 12 & 10.3 & 10.3 & 38.5 \\
\hline & B- & 5 & 4.3 & 4.3 & 42.7 \\
\hline & $\mathrm{B}+$ & 20 & 17.1 & 17.1 & 59.8 \\
\hline & O- & 4 & 3.4 & 3.4 & 63.2 \\
\hline & $\mathrm{O}+$ & 43 & 36.8 & 36.8 & 100.0 \\
\hline & Total & 117 & 100.0 & 100.0 & \\
\hline
\end{tabular}

Blood type: $36.8 \%$ of pregnant women were of $\mathrm{O}+$ blood group.

\section{FBS}

\begin{tabular}{llrrrc}
\hline & Frequency & Percent & Valid Percent & Cumulative Percent \\
\hline \multirow{3}{*}{ Valid } & {$[65-80]$} & 28 & 23.9 & 23.9 & 23.9 \\
\cline { 2 - 6 } & {$[80-120]$} & 78 & 66.7 & 66.7 & 90.6 \\
\cline { 2 - 6 } & {$[120-180]$} & 11 & 9.4 & 9.4 & 100.0 \\
\cline { 2 - 6 } & Total & 117 & 100.0 & 100.0 & \\
\hline
\end{tabular}

\subsection{Inferential Statistics}

\section{Toxoplasmosis infection * age}

\section{Research question: Is age a risk factor of toxoplasmosis?}

$\mathrm{H}_{0}=$ age is not a risk factor of toxoplasmosis.

$\mathrm{Ha}=$ age is a risk factor of toxoplasmosis.

According to the chi-square test, $\mathrm{p}$-value $=0.00$ thus $\mathrm{p}$-value $<0.05$ which implies that it is significant so we reject $\mathrm{H}_{0}$ and accept Ha.

\begin{tabular}{|c|c|c|c|c|c|}
\hline \multicolumn{6}{|l|}{ Crosstab } \\
\hline \multicolumn{6}{|l|}{ Count } \\
\hline & & \multicolumn{3}{|c|}{ age } & \multirow[b]{2}{*}{ Total } \\
\hline & & [20-30] & {$[30-40]$} & {$[40-50]$} & \\
\hline \multirow[t]{2}{*}{ toxoplasmosis infection } & yes & 6 & 4 & 43 & 53 \\
\hline & no & 20 & 27 & 17 & 64 \\
\hline Total & & 26 & 31 & 60 & 117 \\
\hline \multicolumn{6}{|l|}{ Chi-Square Tests } \\
\hline & Value & df & \multicolumn{3}{|c|}{ Asymptotic Significance (2-sided) } \\
\hline Pearson Chi-Square & $35.146^{\mathrm{a}}$ & 2 & \multicolumn{3}{|c|}{.000} \\
\hline Likelihood Ratio & 37.700 & 2 & \multicolumn{3}{|c|}{.000} \\
\hline Linear-by-Linear Association & 24.521 & 1 & \multicolumn{3}{|c|}{.000} \\
\hline $\mathrm{N}$ of Valid Cases & 117 & & & & \\
\hline
\end{tabular}

a. $\quad 0$ cells $(.0 \%)$ have expected count less than 5 . The minimum expected count is 11.78 .

Toxoplasmosis infection * having pets 


\section{Research question: Does having pets increase the chances of being infected with $\boldsymbol{T}$. gondii?}

$\mathrm{H}_{0}=$ having pets doesn't affect the chances of getting infected

$\mathrm{Ha}=$ having pets is a risk factor of toxoplasmosis

According to the chi-square test $\mathrm{p}=0.00<0.05$ so this value is significant and we reject $\mathrm{H}_{0}$ and accept Ha.

\begin{tabular}{|c|c|c|c|c|c|}
\hline Crosstab & & & & & \\
\hline \multicolumn{6}{|l|}{ Count } \\
\hline & & & \multicolumn{2}{|l|}{ pet } & \multirow[b]{2}{*}{ Total } \\
\hline & & & yes & no & \\
\hline \multirow[t]{2}{*}{ toxoplasmosis infection } & \multicolumn{2}{|l|}{ yes } & 43 & 10 & 53 \\
\hline & \multicolumn{2}{|l|}{ no } & 19 & 45 & 64 \\
\hline Total & & & 62 & 55 & 117 \\
\hline \multicolumn{6}{|l|}{ Chi-Square Tests } \\
\hline & Value & $\mathrm{df}$ & $\begin{array}{l}\text { Asymptotic Significance } \\
\text { (2-sided) }\end{array}$ & $\begin{array}{l}\text { Exact Sig. } \\
\text { (2-sided) }\end{array}$ & $\begin{array}{l}\text { Exact Sig. } \\
(1-\text { sided })\end{array}$ \\
\hline Pearson Chi-Square & $30.801^{\mathrm{a}}$ & 1 & .000 & & \\
\hline Continuity Correction $^{\mathrm{b}}$ & 28.771 & 1 & .000 & & \\
\hline Likelihood Ratio & 32.593 & 1 & .000 & & \\
\hline Fisher's Exact Test & & & & .000 & .000 \\
\hline Linear-by-Linear Association & 30.538 & 1 & .000 & & \\
\hline $\mathrm{N}$ of Valid Cases & 117 & & & & \\
\hline
\end{tabular}

a. 0 cells $(.0 \%)$ have expected count less than 5 . The minimum expected count is 24.91 .

b. Computed only for a $2 \times 2$ table.

\section{Toxoplasmosis infection * method of delivery}

\section{Research question: Does toxoplasmosis influence C-section delivery?}

$\mathrm{H}_{0}=$ Toxoplasmosis isn't related to c-section.

$\mathrm{Ha}=$ Toxoplasmosis leads to c-section.

According to the chi-square test $\mathrm{p}=0.00<0.05$ so this value is significant and we reject $\mathrm{H}_{0}$ and accept $\mathrm{Ha}$.

\begin{tabular}{llccc}
\hline Crosstab & & & & \\
\hline Count & & & & \\
& & method of delivery & Total \\
\cline { 2 - 5 } & Normal & c section & 53 \\
\hline $\begin{array}{l}\text { toxoplasmosis } \\
\text { infection }\end{array}$ & no & 4 & 49 & 64 \\
\hline Total & & 25 & 89 & 117 \\
\hline
\end{tabular}

\section{Chi-Square Tests}

\begin{tabular}{lccccc}
\hline & Value & df & $\begin{array}{c}\text { Asymptotic } \\
\text { Significance (2-sided) }\end{array}$ & $\begin{array}{c}\text { Exact Sig. } \\
(2 \text {-sided })\end{array}$ & $\begin{array}{c}\text { Exact Sig. } \\
(1 \text {-sided })\end{array}$ \\
\hline Pearson Chi-Square & $15.446^{\mathrm{a}}$ & 1 & .000 & & \\
\hline Continuity Correction $^{\mathrm{b}}$ & 13.801 & 1 & .000 & .000 & .000 \\
\hline Likelihood Ratio $^{\text {Fisher's Exact Test }}$ & 17.037 & 1 & & .000 \\
\hline
\end{tabular}




\begin{tabular}{llll}
\hline Linear-by-Linear Association & 15.314 & 1 & .000 \\
\hline N of Valid Cases & 117 & & \\
\hline
\end{tabular}

a. 0 cells $(.0 \%)$ have expected count less than 5 . The minimum expected count is 13.14 .

b. Computed only for a $2 \times 2$ table

\section{Toxoplasmosis infection * newborn's health}

\section{Research question: Are newborns of toxoplasmosis diseased mothers also infected?}

$\mathrm{H}_{0}$ : the newborns are healthy

Ha: the newborns are infected

$\mathrm{P}$-value $=0<0.05$ implies the results are significant and we reject $\mathrm{H}_{0}$ and accept $\mathrm{Ha}$.

\begin{tabular}{|c|c|c|c|c|c|}
\hline Crosstab & & & & & \\
\hline \multicolumn{6}{|l|}{ Count } \\
\hline & & \multicolumn{3}{|c|}{ health status of child } & \multirow{2}{*}{ Total } \\
\hline & & normal & infected & & \\
\hline \multirow{2}{*}{$\begin{array}{l}\text { toxoplasmosis } \\
\text { infection }\end{array}$} & yes & 16 & 37 & & 53 \\
\hline & no & 56 & 8 & & 64 \\
\hline Total & & 72 & 45 & & 117 \\
\hline \multicolumn{6}{|l|}{ Chi-Square Tests } \\
\hline & Value & df & $\begin{array}{l}\text { Asymptotic Significance } \\
\text { (2-sided) }\end{array}$ & $\begin{array}{l}\text { Exact Sig. } \\
\text { (2-sided) }\end{array}$ & $\begin{array}{l}\text { Exact Sig. } \\
\text { (1-sided) }\end{array}$ \\
\hline Pearson Chi-Square & $40.233^{\mathrm{a}}$ & 1 & .000 & & \\
\hline Continuity Correction $^{\mathrm{b}}$ & 37.848 & 1 & .000 & & \\
\hline Likelihood Ratio & 42.762 & 1 & .000 & & \\
\hline Fisher's Exact Test & & & & .000 & .000 \\
\hline $\begin{array}{l}\text { Linear-by-Linear } \\
\text { Association }\end{array}$ & 39.889 & 1 & .000 & & \\
\hline $\mathrm{N}$ of Valid Cases & 117 & & & & \\
\hline
\end{tabular}

a. 0 cells $(.0 \%)$ have expected count less than 5 . The minimum expected count is 20.38.

b. Computed only for a $2 \times 2$ table.

\section{Toxoplasmosis infection * body weight}

\section{Research question: Is there a relation between body weight and toxoplasmosis?}

$\mathrm{H}_{0}$ : there isn't any relation between body weight and toxoplasmosis

Ha: there is a correlation between body weight and toxoplasmosis

From chi-square test, $\mathrm{p}$-value $=0.550>0.05$ thus the results aren't significant so we accept $\mathrm{H}_{0}$ and reject $\mathrm{Ha}$. 


\section{Crosstab}

\begin{tabular}{|c|c|c|c|c|c|c|c|}
\hline \multicolumn{8}{|l|}{ Count } \\
\hline & & \multicolumn{5}{|c|}{ body weight } & \multirow[t]{2}{*}{ Total } \\
\hline & & {$[50-65]$} & {$[65=79]$} & [79-94] & [95-109] & [109-135] & \\
\hline \multirow[t]{2}{*}{ toxoplasmosis infection } & yes & 16 & 17 & 13 & 6 & 1 & 53 \\
\hline & no & 17 & 26 & 15 & 3 & 3 & 64 \\
\hline Total & & 33 & 43 & 28 & 9 & 4 & 117 \\
\hline \multicolumn{8}{|l|}{ Chi-Square Tests } \\
\hline & & Value & & $\mathrm{df}$ & \multicolumn{3}{|c|}{ Asymptotic Significance (2-sided) } \\
\hline Pearson Chi-Square & & $3.050^{\mathrm{a}}$ & & 4 & \multicolumn{2}{|c|}{.550} & \\
\hline Likelihood Ratio & & 3.101 & & 4 & \multicolumn{2}{|c|}{.541} & \\
\hline Linear-by-Linear Association & & .014 & & 1 & \multicolumn{2}{|c|}{.905} & \\
\hline $\mathrm{N}$ of Valid Cases & & \multicolumn{5}{|l|}{117} & \\
\hline
\end{tabular}

a. 4 cells $(40.0 \%)$ have expected count less than 5 . The minimum expected count is 1.81 .

\section{Toxoplasmosis * week of delivery}

\section{Research question: Does toxoplasmosis affect the duration of pregnancy?}

$\mathrm{H}_{0}$ : toxoplasmosis doesn't affect the duration of pregnancy

Ha: toxoplasmosis interferes with the duration of pregnancy

From chi-square test, $\mathrm{p}$-value $=0.102>0.05$ therefore the results aren't significant and we accept $\mathrm{H}_{0}$ and reject $\mathrm{Ha}$.

\section{Crosstab}

\begin{tabular}{|c|c|c|c|c|c|}
\hline \multicolumn{6}{|l|}{ Count } \\
\hline & & \multicolumn{3}{|c|}{ week of delivery } & \multirow[b]{2}{*}{ Total } \\
\hline & & {$[30-37]$} & {$[37-40]$} & [40-41] & \\
\hline \multirow[t]{2}{*}{ toxoplasmosis infection } & yes & 7 & 39 & 7 & 53 \\
\hline & no & 3 & 45 & 16 & 64 \\
\hline Total & & 10 & 84 & 23 & 117 \\
\hline \multicolumn{6}{|l|}{ Chi-Square Tests } \\
\hline & \multicolumn{2}{|c|}{ Value } & df & \multicolumn{2}{|c|}{ Asymptotic Significance (2-sided) } \\
\hline Pearson Chi-Square & \multicolumn{2}{|c|}{$4.556^{\mathrm{a}}$} & 2 & \multicolumn{2}{|c|}{.102} \\
\hline Likelihood Ratio & \multicolumn{2}{|c|}{4.656} & 2 & \multicolumn{2}{|c|}{.097} \\
\hline Linear-by-Linear Association & \multicolumn{2}{|c|}{4.397} & 1 & \multicolumn{2}{|c|}{.036} \\
\hline $\mathrm{N}$ of Valid Cases & \multicolumn{2}{|c|}{117} & & & \\
\hline
\end{tabular}

a. 1 cell $(16.7 \%)$ have expected count less than 5 . The minimum expected count is 4.53 . 


\section{Toxoplasmosis infection * residence}

Research question: Is there a difference in the prevalence of the disease among subjects living in a city vs subjects living in a village?

$\mathrm{H}_{0}$ : residence isn't related to the possibility of infection with the parasite T.gondii

Ha: residence affects the chances of infection by $T$. gondii

P-value $=0.479>0.05$ so the results are not significant and $\mathrm{H}_{0}$ is accepted and $\mathrm{Ha}$ is rejected.

\begin{tabular}{|c|c|c|c|c|c|}
\hline Crosstab & & & & & \\
\hline \multicolumn{6}{|l|}{ Count } \\
\hline & & \multicolumn{3}{|c|}{ residence } & \multirow[b]{2}{*}{ Total } \\
\hline & & & city & village & \\
\hline \multirow[t]{2}{*}{ toxoplasmosis infection } & yes & & 19 & 34 & 53 \\
\hline & no & & 19 & 45 & 64 \\
\hline Total & & & 38 & 79 & 117 \\
\hline \multicolumn{6}{|c|}{ Chi-Square Tests } \\
\hline & Value & $\mathrm{df}$ & $\begin{array}{l}\text { Asymptotic Significance } \\
\text { (2-sided) }\end{array}$ & $\begin{array}{l}\text { Exact Sig. } \\
\text { (2-sided) }\end{array}$ & $\begin{array}{l}\text { Exact Sig. } \\
(1-\text { sided })\end{array}$ \\
\hline Pearson Chi-Square & $.502^{\mathrm{a}}$ & 1 & .479 & & \\
\hline Continuity Correction $^{\mathrm{b}}$ & .260 & 1 & .610 & & \\
\hline Likelihood Ratio & .501 & 1 & .479 & & \\
\hline Fisher's Exact Test & & & & .553 & .305 \\
\hline Linear-by-Linear Association & .498 & 1 & .481 & & \\
\hline $\mathrm{N}$ of Valid Cases & 117 & & & & \\
\hline
\end{tabular}

a. 0 cells $(.0 \%)$ have expected count less than 5 . The minimum expected count is 17.21.

b. Computed only for a $2 \times 2$ table.

\section{Toxoplasmosis infection * family history}

Research question: Is family history a risk factor of toxoplasmosis?

$\mathrm{H}_{0}$ : the family history isn't a risk factor of toxoplasmosis

Ha: a family history of toxoplasmosis displays a correlation with the infection of the pregnant women

P-value $=0.213>0.05$ then the results aren't significant and $\mathrm{H}_{0}$ is accepted and $\mathrm{Ha}$ is rejected.

\section{Crosstab}

\section{Count}

\begin{tabular}{lcccc}
\hline & & \multicolumn{2}{c}{ family history } & \\
\cline { 3 - 5 } & & yes & no & Total \\
\hline toxoplasmosis infection & yes & 17 & 36 & 53 \\
\cline { 2 - 5 } & no & 14 & 50 & 64 \\
\hline Total & & 31 & 86 & 117 \\
\hline
\end{tabular}

Chi-Square Tests

\begin{tabular}{lccccc}
\hline & Value & df & $\begin{array}{c}\text { Asymptotic Significance } \\
(2 \text {-sided })\end{array}$ & $\begin{array}{c}\text { Exact Sig. } \\
(2 \text {-sided })\end{array}$ & $\begin{array}{c}\text { Exact Sig. } \\
(1 \text {-sided })\end{array}$ \\
\hline Pearson Chi-Square & $1.549^{\mathrm{a}}$ & 1 & .213 & \\
\hline Continuity Correction $^{\mathrm{b}}$ & 1.069 & 1 & .301 & \\
\hline
\end{tabular}




\begin{tabular}{llllll}
\hline Likelihood Ratio & 1.544 & 1 & .214 & .293 & .151 \\
\hline Fisher's Exact Test & & & & .215 & \\
\hline Linear-by-Linear Association & 1.536 & 1 & &
\end{tabular}

a. 0 cells $(.0 \%)$ have expected count less than 5 . The minimum expected count is 14.04.

b. Computed only for a $2 \times 2$ table.

\section{Toxoplasmosis infection * rubella infection}

Research question: If a pregnant woman has rubella, would that influence the infection by $T$. gondii?

$\mathrm{H}_{0}$ : there's no association between rubella infection and toxoplasmosis

Ha: rubella infection influences toxoplasmosis

$\mathrm{P}$-value $=0.218>0.05$ thus the results are not significant so $\mathrm{H}_{0}$ is accepted and $\mathrm{Ha}$ is rejected.

\begin{tabular}{|c|c|c|c|c|c|}
\hline \multicolumn{6}{|l|}{ Crosstab } \\
\hline \multicolumn{6}{|l|}{ Count } \\
\hline & & & \multicolumn{2}{|c|}{ rubella infection } & \multirow[b]{2}{*}{ Total } \\
\hline & & & yes & no & \\
\hline \multirow{2}{*}{\multicolumn{2}{|c|}{ toxoplasmosis infection }} & yes & 37 & 16 & 53 \\
\hline & & no & 51 & 13 & 64 \\
\hline Total & & & 88 & 29 & 117 \\
\hline \multicolumn{6}{|l|}{ Chi-Square Tests } \\
\hline & Value & df & $\begin{array}{c}\text { Asymptotic Significance } \\
\text { (2-sided) }\end{array}$ & Exact Sig. (2-sided) & $\begin{array}{l}\text { Exact Sig. } \\
\text { (1-sided) }\end{array}$ \\
\hline Pearson Chi-Square & $1.517^{\mathrm{a}}$ & 1 & .218 & & \\
\hline Continuity Correction $^{\mathrm{b}}$ & 1.033 & 1 & .309 & & \\
\hline Likelihood Ratio & 1.512 & 1 & .219 & & \\
\hline Fisher's Exact Test & & & & .283 & .155 \\
\hline $\begin{array}{l}\text { Linear-by-Linear } \\
\text { Association }\end{array}$ & 1.504 & 1 & .220 & & \\
\hline $\mathrm{N}$ of Valid Cases & 117 & & & & \\
\hline
\end{tabular}

a. 0 cells $(.0 \%)$ have expected count less than 5 . The minimum expected count is 13.14 .

b. Computed only for a $2 \times 2$ table.

\section{Toxoplasmosis infection * Hepatitis B surface antigen}

Research question: Is there a relation between infection with hepatitis $B$ virus and toxoplasmosis?

$\mathrm{H}_{0}$ : HBsAg and toxoplasmosis seropositivity are independent

Ha: There is a relation between hepatitis $\mathrm{B}$ and toxoplasmosis

$\mathrm{P}$-value $=0.127>0.05$ so results aren't significant hence $\mathrm{H}_{0}$ is accepted and $\mathrm{Ha}$ is rejected. 


\section{Crosstab}

Count

\begin{tabular}{|c|c|c|c|c|c|c|}
\hline & & & & \multicolumn{2}{|l|}{ Hbs Ag } & \multirow[b]{2}{*}{ Total } \\
\hline & & & & yes & no & \\
\hline \multirow[t]{2}{*}{ Toxoplasmosis infection } & & & yes & 43 & 10 & 53 \\
\hline & & & no & 44 & 20 & 64 \\
\hline Total & & & & 87 & 30 & 117 \\
\hline \multicolumn{7}{|l|}{ Chi-Square Tests } \\
\hline & Value & $\mathrm{df}$ & $\begin{array}{c}\text { Asymptotic } \\
\text { Significance (2-sided) }\end{array}$ & Exact Sig. (2-sided) & & $\begin{array}{l}\text { Exact Sig. } \\
(1-\text { sided })\end{array}$ \\
\hline Pearson Chi-Square & $2.331^{\mathrm{a}}$ & 1 & .127 & & & \\
\hline Continuity Correction $^{\mathrm{b}}$ & 1.727 & 1 & .189 & & & \\
\hline Likelihood Ratio & 2.374 & 1 & .123 & & & \\
\hline Fisher's Exact Test & & & & .142 & & .094 \\
\hline Linear-by-Linear Association & 2.311 & 1 & .128 & & & \\
\hline $\mathrm{N}$ of Valid Cases & 117 & & & & & \\
\hline
\end{tabular}

a. 0 cells $(.0 \%)$ have expected count less than 5 . The minimum expected count is 13.59 .

b. Computed only for a $2 \times 2$ table

\section{Toxoplasmosis infection * Hemoglobin}

\section{Research question: Is hemoglobin level correlated with the occurrence of toxoplasmosis?}

$\mathrm{H}_{0}$ : Hemoglobin level doesn't show any relation with toxoplasmosis

$\mathrm{Ha}: \mathrm{Hb}$ level shows a significant relation with toxoplasmosis

$\mathrm{P}$-value $=0.494>0.05$ therefore the results aren't significant so $\mathrm{H}_{0}$ is accepted and Ha is rejected.

\section{Crosstab}

Count

\begin{tabular}{lcccc}
\hline & & \multicolumn{3}{c}{ Hbg } \\
\cline { 3 - 5 } & & {$[7-10]$} & {$[10-14]$} & Total \\
\hline Toxoplasmosis infection & yes & 9 & 44 & 53 \\
\cline { 2 - 5 } & no & 8 & 56 & 64 \\
\hline Total & & 17 & 100 & 117 \\
\hline
\end{tabular}

Chi-Square Tests

\begin{tabular}{lllccc}
\hline & Value & df & $\begin{array}{c}\text { Asymptotic Significance } \\
(2 \text {-sided })\end{array}$ & $\begin{array}{c}\text { Exact Sig. } \\
(2 \text {-sided })\end{array}$ & $\begin{array}{c}\text { Exact Sig. } \\
(1 \text {-sided })\end{array}$ \\
\hline Pearson Chi-Square & $.469^{\mathrm{a}}$ & 1 & .494 & .674 & .601 \\
\hline Continuity Correction $^{\mathrm{b}}$ & .177 & 1 & .495 & .336 \\
\hline Likelihood Ratio & .467 & 1 & & .495 & \\
\hline Fisher's Exact Test & & & &
\end{tabular}

a. 0 cells $(.0 \%)$ have expected count less than 5 . The minimum expected count is 7.70 .

b. Computed only for a $2 \times 2$ table. 


\section{Toxoplasmosis infection * Vitamin D3 level}

Research question: Is there an association between vitamin D3 level and toxoplasmosis?

$\mathrm{H}_{0}$ : Vitamin D3 level doesn't relate to toxoplasmosis

Ha: there's a relation between vitamin D3 level and toxoplasmosis

$\mathrm{P}$-value $=0.37>0.05$ so the results aren't significant thus $\mathrm{H}_{0}$ is accepted and Ha is rejected.

\begin{tabular}{|c|c|c|c|c|c|c|}
\hline \multicolumn{7}{|l|}{ Crosstab } \\
\hline \multicolumn{7}{|l|}{ Count } \\
\hline & & & \multicolumn{3}{|l|}{ Vitamin D3 level } & \multirow{2}{*}{ Total } \\
\hline & & & deficiency & normal & & \\
\hline \multirow{2}{*}{ Toxoplasmosis infection } & & yes & 47 & 6 & & 53 \\
\hline & & no & 53 & 11 & & 64 \\
\hline Total & & & 100 & 17 & & 117 \\
\hline \multicolumn{7}{|l|}{ Chi-Square Tests } \\
\hline & Value & $\mathrm{df}$ & $\begin{array}{l}\text { Asymptotic } \\
\text { Significance (2-sided) }\end{array}$ & $\begin{array}{l}\text { Exact } \\
\text { (2-sided) }\end{array}$ & Sig. & $\begin{array}{l}\text { Exact Sig. } \\
(1 \text {-sided })\end{array}$ \\
\hline Pearson Chi-Square & $.804^{\mathrm{a}}$ & 1 & .370 & & & \\
\hline Continuity Correction $^{\mathrm{b}}$ & .401 & 1 & .527 & & & \\
\hline Likelihood Ratio & .817 & 1 & .366 & & & \\
\hline Fisher's Exact Test & & & & .437 & & .265 \\
\hline $\begin{array}{l}\text { Linear-by-Linear } \\
\text { Association }\end{array}$ & .797 & 1 & .372 & & & \\
\hline $\mathrm{N}$ of Valid Cases & 117 & & & & & \\
\hline
\end{tabular}

a. 0 cells $(.0 \%)$ have expected count less than 5 . The minimum expected count is 7.70 .

b. Computed only for a $2 \times 2$ table.

\section{Toxoplasmosis infection * TSH level}

Research question: Is TSH level a parameter that relates to toxoplasmosis?

$\mathrm{H}_{0}$ : TSH level and toxoplasmosis are independent of each other

Ha: TSH level and toxoplasmosis are correlated

P-value $=0.194>0.05$ thus the results aren't significant and $\mathrm{H}_{0}$ is accepted while Ha is rejected.

\begin{tabular}{|c|c|c|c|c|c|c|}
\hline Crosstab & & & & & & \\
\hline \multicolumn{7}{|l|}{ Count } \\
\hline & & \multicolumn{4}{|c|}{ TSH level } & \multirow[b]{2}{*}{ Total } \\
\hline & & & normal & deficien & & \\
\hline \multirow[t]{2}{*}{ toxoplasmosis infection } & yes & & 53 & 0 & & 53 \\
\hline & no & & 62 & 2 & & 64 \\
\hline Total & & & 115 & 2 & & 117 \\
\hline \multicolumn{7}{|l|}{ Chi-Square Tests } \\
\hline & & Value & df & $\begin{array}{c}\text { Asymptotic } \\
\text { Significance (2-sided) }\end{array}$ & $\begin{array}{l}\text { Exact Sig. } \\
\text { (2-sided) }\end{array}$ & $\begin{array}{l}\text { Exact Sig. } \\
\text { (1-sided) }\end{array}$ \\
\hline Pearson Chi-Square & & $1.685^{\mathrm{a}}$ & 1 & .194 & & \\
\hline Continuity Correction $^{\mathrm{b}}$ & & .338 & 1 & .561 & & \\
\hline
\end{tabular}




\begin{tabular}{llllll}
\hline Likelihood Ratio & 2.442 & 1 & .118 & .500 & .297 \\
\hline Fisher's Exact Test & & & .196 & \\
\hline Linear-by-Linear Association & 1.671 & 1 & & \\
\hline N of Valid Cases & 117 & &
\end{tabular}

a. 2 cells $(50.0 \%)$ have expected count less than 5. The minimum expected count is .91 .

b. Computed only for a $2 \times 2$ table.

\section{Toxoplasmosis * gender}

\section{Research question: Does $\boldsymbol{T}$. gondii infect a certain gender more than the other?}

$\mathrm{H}_{0}$ : Gender doesn't affect toxoplasmosis occurrence

Ha: Toxoplasmosis is gender biased

$\mathrm{P}$-value $=0.691>0.05$ so results aren't significant thus $\mathrm{H}_{0}$ is accepted whereas $\mathrm{Ha}$ is rejected

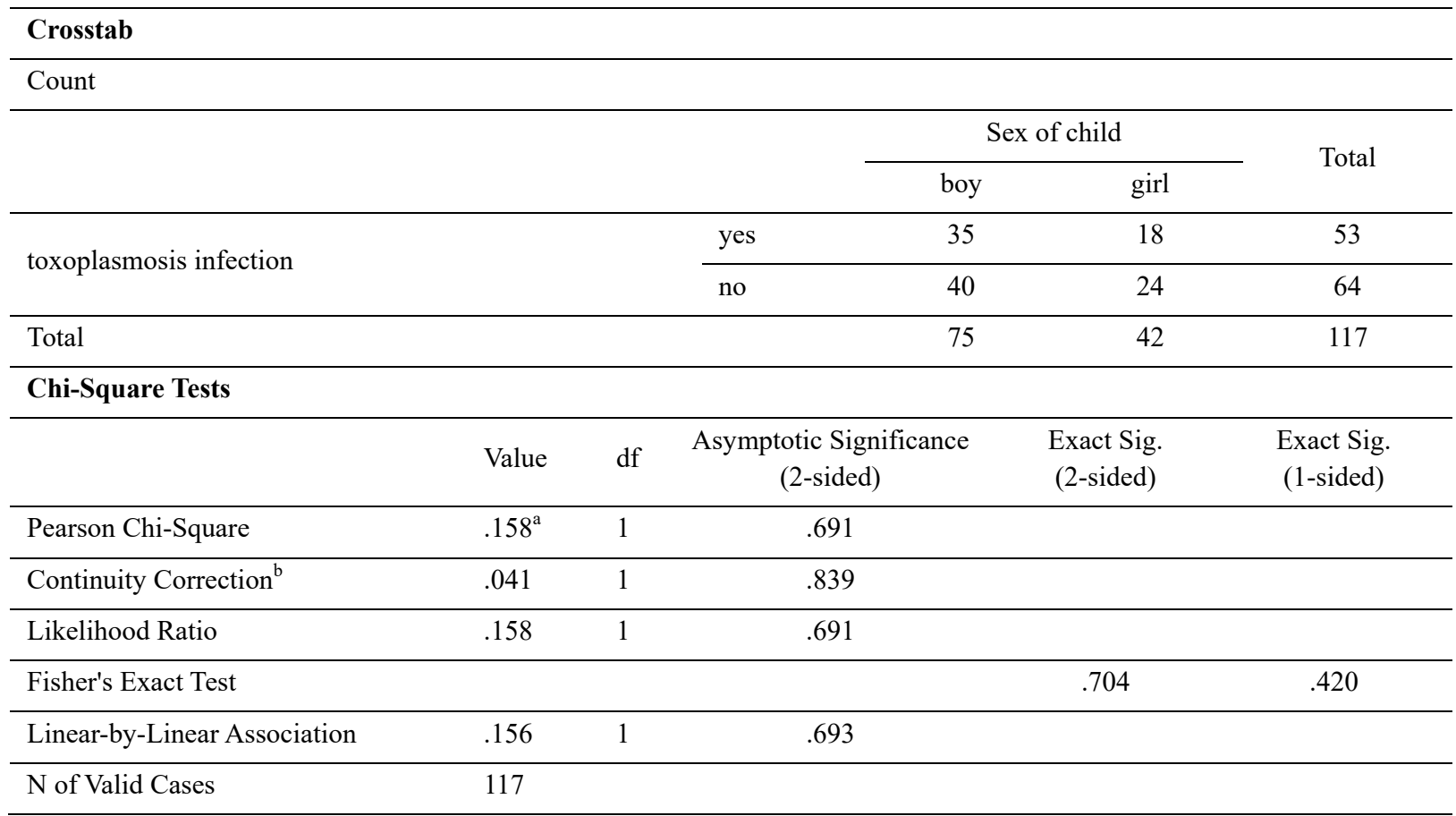

a. 0 cells $(.0 \%)$ have expected count less than 5 . The minimum expected count is 19.03.

b. Computed only for a $2 \times 2$ table. 


\section{Toxoplasmosis * blood type}

Research question: Does having a certain blood type boost the chances of toxoplasmosis?

\section{Crosstab}

\begin{tabular}{|c|c|c|c|c|c|c|c|c|c|c|}
\hline \multicolumn{11}{|c|}{ Count } \\
\hline & & \multicolumn{8}{|c|}{ blood type } & \multirow{2}{*}{ Total } \\
\hline & & A- & $\mathrm{A}+$ & AB- & $\mathrm{AB}+$ & B- & $\mathrm{B}+$ & O- & $\mathrm{O}+$ & \\
\hline \multirow{2}{*}{ toxoplasmosis infection } & yes & 0 & 11 & 0 & 4 & 2 & 11 & 1 & 24 & 53 \\
\hline & no & 1 & 20 & 1 & 8 & 3 & 9 & 3 & 19 & 64 \\
\hline Total & & 1 & 31 & 1 & 12 & 5 & 20 & 4 & 43 & 117 \\
\hline
\end{tabular}

Chi-Square Tests

\begin{tabular}{cccc}
\hline & Value & df & Asymptotic Significance (2-sided) \\
\hline Pearson Chi-Square & $6.955^{\text {a }}$ & 7 & .434 \\
\hline Likelihood Ratio & 7.778 & 7 & .353 \\
\hline Linear-by-Linear Association & 4.123 & 1 & .042 \\
\hline N of Valid Cases & 117 & & \\
\hline
\end{tabular}

$\mathrm{H}_{0}$ : Different blood types don't affect the likelihood of toxoplasmosis

Ha: Blood types could influence the possibility of toxoplasmosis

$\mathrm{P}$-value $=0.434>0.05$ so results aren't significant which implies that $\mathrm{H}_{0}$ is accepted and $\mathrm{Ha}$ is rejected.

\begin{tabular}{|c|c|c|c|c|c|}
\hline Crosstab & & & & & \\
\hline \multicolumn{6}{|l|}{ Count } \\
\hline & & \multicolumn{3}{|l|}{ FBS } & \multirow{2}{*}{ Total } \\
\hline & & {$[65-80]$} & [80-120] & {$[120-180]$} & \\
\hline \multirow{2}{*}{ Toxoplasmosis infection } & yes & 17 & 31 & 5 & 53 \\
\hline & no & 11 & 47 & 6 & 64 \\
\hline Total & & 28 & 78 & 11 & 117 \\
\hline
\end{tabular}

a. 8 cells $(50.0 \%)$ have expected count less than 5 . The minimum expected count is .45 .

\section{Toxoplasmosis * Fasting blood sugar}

\section{Research question: Is fasting blood sugar a parameter related to toxoplasmosis?}

$\mathrm{H}_{0}$ : there's no link between FBS and toxoplasmosis

Ha: there's a connection between FBS and toxoplasmosis

$\mathrm{P}$-value $=0.161>0.05$ hence the results aren't significant so $\mathrm{H}_{0}$ is accepted and $\mathrm{Ha}$ is rejected.

\section{Chi-Square Tests}

\begin{tabular}{lllc}
\hline & Value & df & Asymptotic Significance (2-sided) \\
\hline Pearson Chi-Square & $3.657^{\mathrm{a}}$ & 2 & .161 \\
Likelihood Ratio & 3.657 & 2 & .161 \\
Linear-by-Linear Association & 2.024 & 1 & .155 \\
N of Valid Cases & 117 & & \\
\hline
\end{tabular}

a. 1 cells $(16.7 \%)$ have expected count less than 5 . The minimum expected count is 4.98 . 


\section{Discussion}

The significant results in our studied sample: age, having pets, mode of delivery and the health status of the fetus were congruous with previous studies in different populations. In our findings, the older the pregnant women, the more the observed cases of toxoplasmosis. Also, the infection was shown to be related to owning pets. As for the mode of delivery, a great percentage of pregnant women having toxoplasmosis gave birth through a caesarean section. Out of these infected women, a significant number delivered babies infected with $T$. gondii. The rest of the studied variables weren't significant for our chosen population although some of these results aren't consistent with previous studies in other populations. For example, residence was a significant parameter in other studies while in ours it wasn't due to the fact that some Lebanese villages are turning into city like areas with limited gardening and farming. In addition, we investigated some biological and hormonal parameters that are important in any pregnancy but none showed any correlation with toxoplasmosis infection and transmission.

\section{Conclusion}

Testing for Toxoplasma gondii before pregnancy via a blood test is a must since there is a chance for newborns to get infected. However, if being positively diagnosed with toxoplasmosis after pregnancy, several medications can be used for treatments. Precaution measurements are a necessity to avoid future infections during pregnancy. In Chouf and Saida, infection of pregnant women with T.gondii is correlated to their age and exposure to pets. The infection of the pregnant ladies leads to caesarean section delivery and to toxoplasmosis infected newborns.

\section{Acknowledgements}

We would like to thank the gynecologists that cooperated with us, Dr. Tatiana Bou Orm, Dr. Alia Chebbo and Dr. Bouchra El-Hajj. The study couldn't have been done without the data they offered. A special thanks to Dr. Tatiana for the speed in giving us an appointment in which she had the files prepared for us.

\section{Competing Interests Statement}

The authors declare that there are no competing or potential conflicts of interest.

\section{References}

Jones, J. L., Kruszon-Moran, D., Wilson, M., McQuillan, G., Navin, T., \& McAuley, J. B. (2001). Toxoplasma gondii infection in the United States: seroprevalence and risk factors. American Journal of Epidemiology, 154(4), 357-365. https://doi.org/10.1093/aje/154.4.357

Guerina, N. G., \& Lee, J. (2017). Congenital toxoplasmose: treatment, outcome, and prevention. Retrieved from $\mathrm{http} / /$ www.update.com/contents/congenital-toxoplasmosis-treatment-outcome-and-prevention?source=see link\#H4829752

Hill, D., \& Dubey, J. P. (2002). Toxoplasma gondii, "transmission, diagnosis and prevention". Clin Microbiol Infect, 8, 634-640. https://doi.org/10.1046/j.1469-0691.2002.00485.x

Bernsteen, L., Gregory, C. R., Aronson, L. R., Lirtzman, R. A., \& Brummer, D. G. (1999). Acute toxoplasmosis following renal transplantation in three cats and a dog. J Am Vet Med Assoc, 215, 1123-6.

Smith, J. L. (1999). Foodborne infections during pregnancy. $J$ Food Protection, 62, 818-29. https://doi.org/10.4315/0362-028X-62.7.818

Dunn, D., Wallon, M., Peyron, F., Petersen, E., Peckham, C., \& Gilbert, R. (1999). Mother-to-child transmission of toxoplasmosis: risk estimates for clinical counselling. Lancet, 353, 1829-33. https://doi.org/10.1016/S0140-6736(98)08220-8

Soares, J. A. S., \& Caldeira, A. P. (2019). Congenital toxoplasmosis: the challenge of early diagnosis of a complex and neglected disease. Rev Soc Bras Med Trop., 52, e20180228. https://doi.org/10.1590/0037-8682-0228-2018

Olariu, T. R., Press, C., Talucod, J., Olson, K., \& Montoya, J. G. (2019). Congenital toxoplasmosis in the United States: clinical and serologic findings in infants born to mothers treated during pregnancy. Parasite, 26, 13. https://doi.org/10.1051/parasite/2019013

\section{Copyrights}

Copyright for this article is retained by the author(s), with first publication rights granted to the journal.

This is an open-access article distributed under the terms and conditions of the Creative Commons Attribution license (http://creativecommons.org/licenses/by/4.0/). 